\title{
EVRNet: Efficient Video Restoration on Edge Devices - Supplementary
}

\author{
Sachin Mehta* \\ University of Washington \\ USA \\ Varun Nasery \\ Facebook Inc. \\ USA
}

\author{
Amit Kumar \\ Facebook Inc. \\ USA \\ Vikram Mulukutla \\ Facebook Inc. \\ USA \\ Vikas Chandra \\ Facebook Inc.
}

\author{
Fitsum $\operatorname{Reda}^{\dagger}$ \\ Google \\ USA \\ Rakesh Ranjan \\ Facebook Inc. \\ USA
}

\begin{abstract}
ACM Reference Format:
Sachin Mehta, Amit Kumar, Fitsum Reda, Varun Nasery, Vikram Mulukutla, Rakesh Ranjan, and Vikas Chandra. 2021. EVRNet: Efficient Video Restoration on Edge Devices - Supplementary. In Proceedings of the 29th ACM International Conference on Multimedia (MM '21), October 20-24, 2021, Virtual Event, China. ACM, New York, NY, USA, 12 pages. https://doi.org/10. $1145 / 3474085.3475477$
\end{abstract}

\section{ABLATIONS}

Effect of different CUs: Table 1 studies the effect of single- and multi-scale convolutional units (CUs) with and without SE unit. Multi-scale CU units with SE help improve the performance in case of AWGN denoising while no gain was observed in case of deblocking and super-resolution. We hypothesize that this is because compression happens at macro-block level, and both single and multi-scale blocks are able to effectively remove compression artifacts. Unlike macro-block compression, AWGN noise is identically distributed in the frames and kernels at different scales helps learn better representations and remove noisy artifacts (see gray color row in Table $1 \mathrm{~b}$ ).

Effect of the depth of alignment, differential, and fusion modules: Table 2 studies EVRNet with different values of $N_{A}, N_{D}$, and $N_{F}$. We are interested in efficient networks for edge devices, therefore, we studied only those combinations that satisfies this criteria: $N_{A}+N_{D}+N_{F}=9$. Similar to the effect of different CUs, we did not observe much gains when varying the depth of alignment, differential, and fusion modules for the task of deblocking and super-resolution. However, for denoising, we found that deeper

\footnotetext{
*Work completed during internship at Facebook Inc..

${ }^{\dagger}$ Work done while working at Facebook Inc.

Permission to make digital or hard copies of all or part of this work for personal or classroom use is granted without fee provided that copies are not made or distributed for profit or commercial advantage and that copies bear this notice and the full citation on the first page. Copyrights for components of this work owned by others than ACM must be honored. Abstracting with credit is permitted. To copy otherwise, or republish, to post on servers or to redistribute to lists, requires prior specific permission and/or a fee. Request permissions from permissions@acm.org.

MM '21, October 20-24, 2021, Virtual Event, China

(C) 2021 Association for Computing Machinery.

ACM ISBN 978-1-4503-8651-7/21/10 .\$15.00

https://doi.org/10.1145/3474085.3475477
}

alignment modules delivers the best trade-off between performance and MACs. Therefore, in our main experiments, we used $N_{A}=5$, $N_{D}=2$, and $N_{F}=2$ (see gray color row in Table 2).

\section{QUALITATIVE RESULTS ON THE VIMEO-90K DATASET}

\subsection{Deblocking}

Figures 1, 2, and 3 demonstrate EVRNet's ability in deblocking videos at different compression factors in diverse environments $(Q$; lower value of $Q$ means higher compression). For example, in Figure 1b, EVRNet is able to remove the macro-block artifacts even under high compression $(Q=15)$ around objects (e.g., hand, vegetables, and mixing bowl).

\subsection{Denoising}

Figures 4, 5, 6, 7, and 8 demonstrates EVRNet's ability in denoising different types of noise in diverse scenes. For example, in Figure $4 \mathrm{c}$, EVRNet is able to remove the noise and restore videos with high-quality.

\section{$2.3 \quad$ Video super-resolution $(4 \times)$}

Figure 9 and 10 shows that EVRNet is effective in restoring the details for $4 \times$ video super-resolution in diverse settings. For example, in Figure 10a, EVRNet is able to restore fine details (e.g., hair strands) which are hard to restore with bicubic interpolation. 


\begin{tabular}{lccccccccc}
\hline & & & & \multicolumn{2}{c}{ RGB } & & \multicolumn{2}{c}{ Y-Channel } \\
CU Type & SE Unit & MACs & \# Params & PSNR & SSIM & & PSNR & SSIM \\
\hline Single & $\boldsymbol{X}$ & $9.85 \mathrm{G}$ & $68.15 \mathrm{~K}$ & 36.358 & 0.948 & & 38.477 & 0.961 \\
Single & $\checkmark$ & $9.85 \mathrm{G}$ & $72.95 \mathrm{~K}$ & 36.323 & 0.948 & & 38.403 & 0.961 \\
\hline Multi & $\boldsymbol{X}$ & $10.79 \mathrm{G}$ & $73.91 \mathrm{~K}$ & 36.297 & 0.947 & & 38.363 & 0.961 \\
Multi & $\checkmark$ & $10.79 \mathrm{G}$ & $78.71 \mathrm{~K}$ & 36.334 & 0.948 & & 38.478 & 0.962 \\
\hline
\end{tabular}

(a) Deblocking $(Q=40)$

\begin{tabular}{|c|c|c|c|c|c|c|c|}
\hline \multirow[b]{2}{*}{ CU Type } & \multirow[b]{2}{*}{ SE Unit } & \multirow[b]{2}{*}{ MACs } & \multirow[b]{2}{*}{ \# Params } & \multicolumn{2}{|c|}{ RGB } & \multicolumn{2}{|c|}{ Y-Channel } \\
\hline & & & & PSNR & $\overline{\text { SSIM }}$ & $\begin{array}{l}\text { PSNR } \\
\end{array}$ & SSIM \\
\hline Single & $x$ & $9.85 \mathrm{G}$ & $68.15 \mathrm{~K}$ & 31.207 & 0.868 & 32.650 & 0.886 \\
\hline Single & $\checkmark$ & $9.85 \mathrm{G}$ & $72.95 \mathrm{~K}$ & 32.006 & 0.896 & 33.365 & 0.914 \\
\hline Multi & $x$ & $10.79 \mathrm{G}$ & $73.91 \mathrm{~K}$ & 29.026 & 0.875 & 30.247 & 0.895 \\
\hline Multi & $\checkmark$ & $10.79 \mathrm{G}$ & $78.71 \mathrm{~K}$ & 32.370 & 0.900 & 33.679 & 0.916 \\
\hline
\end{tabular}

(b) AWGN Denoising $\left(\sigma^{2}=0.001\right)$

\begin{tabular}{|c|c|c|c|c|c|c|c|}
\hline \multirow[b]{2}{*}{ CU Type } & \multirow[b]{2}{*}{ SE Unit } & \multirow[b]{2}{*}{ MACs } & \multirow[b]{2}{*}{ \# Params } & \multicolumn{2}{|c|}{ RGB } & \multicolumn{2}{|c|}{ Y-Channel } \\
\hline & & & & PSNR & SSIM & PSNR & SSIM \\
\hline Single & $x$ & $9.90 \mathrm{G}$ & $68.33 \mathrm{~K}$ & 37.406 & 0.962 & 38.042 & 0.966 \\
\hline Single & $\checkmark$ & $9.90 \mathrm{G}$ & $73.14 \mathrm{~K}$ & 37.318 & 0.962 & 37.955 & 0.965 \\
\hline Multi & $x$ & $10.84 \mathrm{G}$ & $74.10 \mathrm{~K}$ & 37.181 & 0.962 & 37.868 & 0.966 \\
\hline Multi & $\checkmark$ & $10.84 \mathrm{G}$ & $78.91 \mathrm{~K}$ & 37.378 & 0.962 & 38.002 & 0.966 \\
\hline
\end{tabular}

(c) Super-resolution $(2 \times)$

Table 1: Effect of different CU units. Multi-scale blocks are effective in restoring fine-grained details (e.g., noise) while both single- and multi-scale blocks are effective in restoring block-level artifacts (e.g., compression). Here, we used $N_{A}=$ $N_{D}=N_{F}=3$.

\begin{tabular}{|c|c|c|c|c|c|c|c|c|}
\hline \multicolumn{3}{|c|}{ Module depth } & \multirow[b]{2}{*}{ MACs } & \multirow[b]{2}{*}{ \# Params } & \multicolumn{2}{|c|}{ RGB } & \multicolumn{2}{|c|}{ Y-Channel } \\
\hline$N_{A}$ & $N_{D}$ & $N_{F}$ & & & PSNR & SSIM & PSNR & SSIM \\
\hline 1 & 1 & 7 & $11.44 \mathrm{G}$ & $78.71 \mathrm{~K}$ & 36.320 & 0.948 & 38.411 & 0.961 \\
\hline 1 & 7 & 1 & $11.44 \mathrm{G}$ & $78.71 \mathrm{~K}$ & 36.356 & 0.948 & 38.450 & 0.962 \\
\hline 7 & 1 & 1 & $9.47 \mathrm{G}$ & $78.71 \mathrm{~K}$ & 36.334 & 0.948 & 38.472 & 0.961 \\
\hline 2 & 2 & 5 & $11.11 \mathrm{G}$ & $78.71 \mathrm{~K}$ & 36.200 & 0.946 & 38.297 & 0.960 \\
\hline 2 & 5 & 2 & $11.11 \mathrm{G}$ & $78.71 \mathrm{~K}$ & 36.327 & 0.948 & 38.412 & 0.962 \\
\hline 5 & 2 & 2 & $10.13 \mathrm{G}$ & $78.71 \mathrm{~K}$ & 36.307 & 0.947 & 38.403 & 0.961 \\
\hline 3 & 2 & 4 & $10.77 \mathrm{G}$ & $78.71 \mathrm{~K}$ & 36.359 & 0.948 & 38.451 & 0.962 \\
\hline 3 & 4 & 2 & $10.77 \mathrm{G}$ & $78.71 \mathrm{~K}$ & 36.307 & 0.947 & 38.390 & 0.961 \\
\hline 4 & 3 & 2 & $10.46 \mathrm{G}$ & $78.71 \mathrm{~K}$ & 36.287 & 0.948 & 38.405 & 0.961 \\
\hline 3 & 3 & 3 & $10.79 \mathrm{G}$ & $78.71 \mathrm{~K}$ & 36.334 & 0.948 & 38.478 & 0.962 \\
\hline
\end{tabular}

(a) Deblocking $(Q=40)$

\begin{tabular}{|c|c|c|c|c|c|c|c|c|}
\hline \multicolumn{3}{|c|}{ Module depth } & \multirow[b]{2}{*}{ MACs } & \multirow[b]{2}{*}{ \# Params } & \multicolumn{2}{|c|}{ RGB } & \multicolumn{2}{|c|}{ Y-Channel } \\
\hline$N_{A}$ & $N_{D}$ & $N_{F}$ & & & PSNR & SSIM & PSNR & SSIM \\
\hline 1 & 1 & 7 & $11.44 \mathrm{G}$ & $78.71 \mathrm{~K}$ & 31.605 & 0.887 & 32.913 & 0.905 \\
\hline 1 & 7 & 1 & $11.44 \mathrm{G}$ & $78.71 \mathrm{~K}$ & 31.753 & 0.884 & 32.951 & 0.901 \\
\hline 7 & 1 & 1 & $9.47 \mathrm{G}$ & $78.71 \mathrm{~K}$ & 30.859 & 0.871 & 32.139 & 0.890 \\
\hline 2 & 2 & 5 & $11.11 \mathrm{G}$ & $78.71 \mathrm{~K}$ & 32.139 & 0.901 & 33.477 & 0.919 \\
\hline 2 & 5 & 2 & $11.11 \mathrm{G}$ & $78.71 \mathrm{~K}$ & 32.057 & 0.891 & 33.445 & 0.908 \\
\hline 5 & 2 & 2 & $10.13 \mathrm{G}$ & $78.71 \mathrm{~K}$ & 32.403 & 0.903 & 33.884 & 0.921 \\
\hline 3 & 2 & 4 & $10.77 \mathrm{G}$ & $78.71 \mathrm{~K}$ & 31.690 & 0.890 & 33.047 & 0.908 \\
\hline 3 & 4 & 2 & $10.77 \mathrm{G}$ & $78.71 \mathrm{~K}$ & 30.785 & 0.874 & 32.193 & 0.896 \\
\hline 4 & 3 & 2 & $10.46 \mathrm{G}$ & $78.71 \mathrm{~K}$ & 31.416 & 0.877 & 32.690 & 0.895 \\
\hline 3 & 3 & 3 & $10.79 \mathrm{G}$ & $78.71 \mathrm{~K}$ & 32.370 & 0.900 & 33.679 & 0.916 \\
\hline
\end{tabular}

(b) AWGN Denoising $\left(\sigma^{2}=0.001\right)$

\begin{tabular}{|c|c|c|c|c|c|c|c|c|}
\hline \multicolumn{3}{|c|}{ Module depth } & \multirow[b]{2}{*}{ MACs } & \multirow[b]{2}{*}{ \# Params } & \multicolumn{2}{|c|}{ RGB } & \multicolumn{2}{|c|}{ Y-Channel } \\
\hline$N_{A}$ & $N_{D}$ & $N_{F}$ & & & PSNR & SSIM & PSNR & SSIM \\
\hline 1 & 1 & 7 & $11.50 \mathrm{G}$ & $78.91 \mathrm{~K}$ & 37.071 & 0.961 & 37.742 & 0.965 \\
\hline 1 & 7 & 1 & $11.50 \mathrm{G}$ & $78.91 \mathrm{~K}$ & 37.136 & 0.961 & 37.774 & 0.965 \\
\hline 7 & 1 & 1 & $9.52 \mathrm{G}$ & $78.91 \mathrm{~K}$ & 37.176 & 0.961 & 37.868 & 0.965 \\
\hline 2 & 2 & 5 & $11.17 \mathrm{G}$ & $78.91 \mathrm{~K}$ & 37.072 & 0.961 & 37.740 & 0.965 \\
\hline 2 & 5 & 2 & $11.17 \mathrm{G}$ & $78.91 \mathrm{~K}$ & 37.102 & 0.961 & 37.776 & 0.965 \\
\hline 5 & 2 & 2 & $10.18 \mathrm{G}$ & $78.91 \mathrm{~K}$ & 37.196 & 0.961 & 37.855 & 0.965 \\
\hline 3 & 2 & 4 & $10.84 \mathrm{G}$ & $78.91 \mathrm{~K}$ & 37.227 & 0.962 & 37.902 & 0.965 \\
\hline 3 & 4 & 2 & $10.84 \mathrm{G}$ & $78.91 \mathrm{~K}$ & 37.071 & 0.961 & 37.740 & 0.965 \\
\hline 4 & 3 & 2 & $10.51 \mathrm{G}$ & $78.91 \mathrm{~K}$ & 37.173 & 0.961 & 37.877 & 0.965 \\
\hline 3 & 3 & 3 & $10.84 \mathrm{G}$ & $78.91 \mathrm{~K}$ & 37.378 & 0.962 & 38.002 & 0.966 \\
\hline
\end{tabular}

(c) Super-resolution $(2 \times)$

Table 2: Effect of the depth of alignment, differential, and fusion modules in the EVRNet. Overall, EVRNet with deeper alignment modules provides the best trade-off between performance and number of multiplication-addition operations (MACs). In all these models, the depth of the network is fixed, i.e., $N_{A}+N_{D}+N_{F}=9$. 


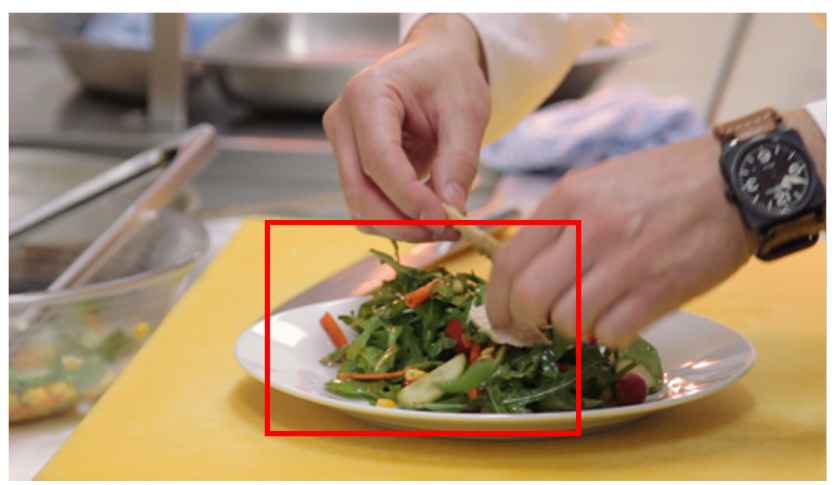

(a) Original
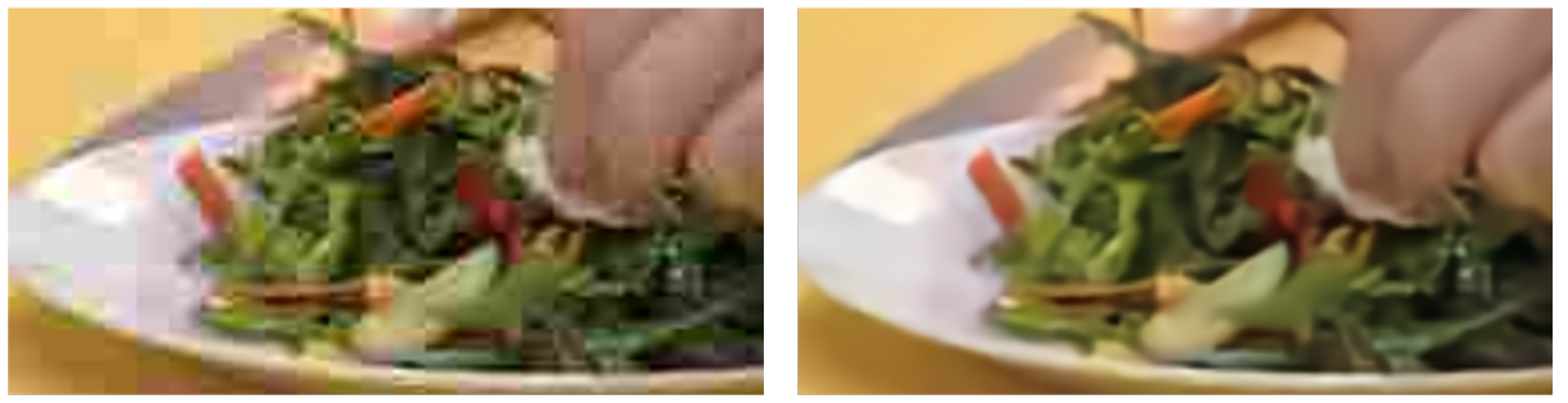

(b) Left: Compressed frame $(Q=15)$. Right: Deblocked image (RGB PSNR: $31.31 \mathrm{~dB})$
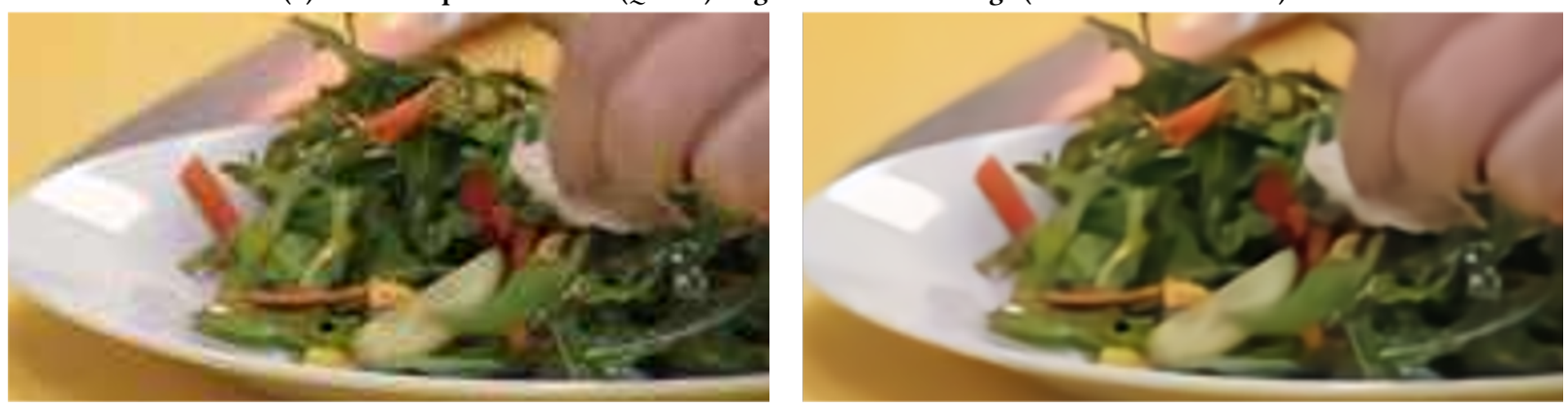

(c) Left: Compressed frame $(Q=45)$. Right: Deblocked image (RGB PSNR: $34.79 \mathrm{~dB})$
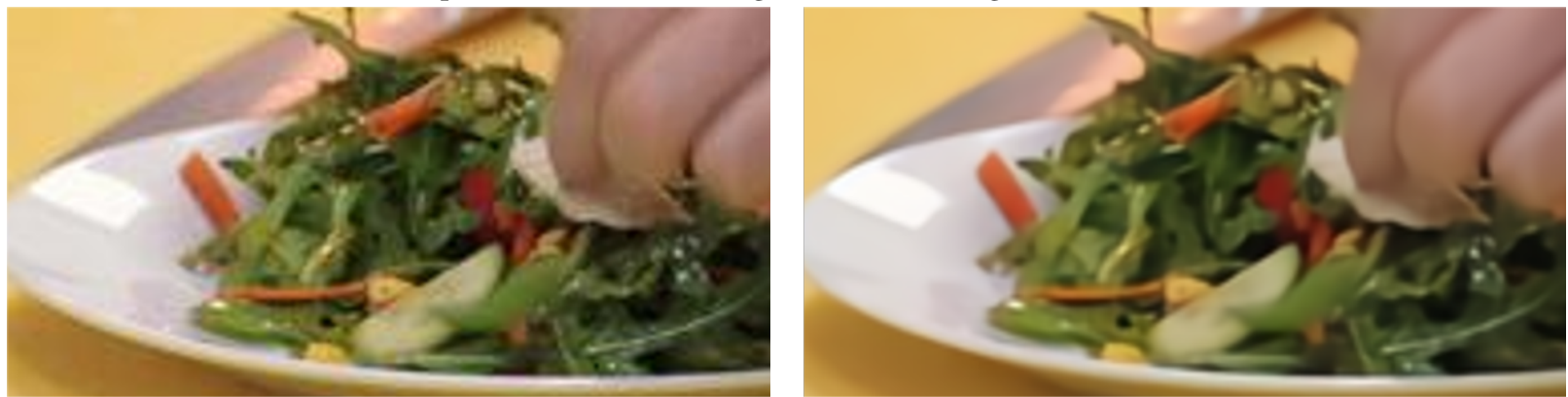

(d) Left: Compressed frame $(Q=75)$. Right: Deblocked image (RGB PSNR: $36.00 \mathrm{~dB})$

Figure 1: Deblocking example at different values of $Q$. Note that lower value of $Q$ means higher compression. 


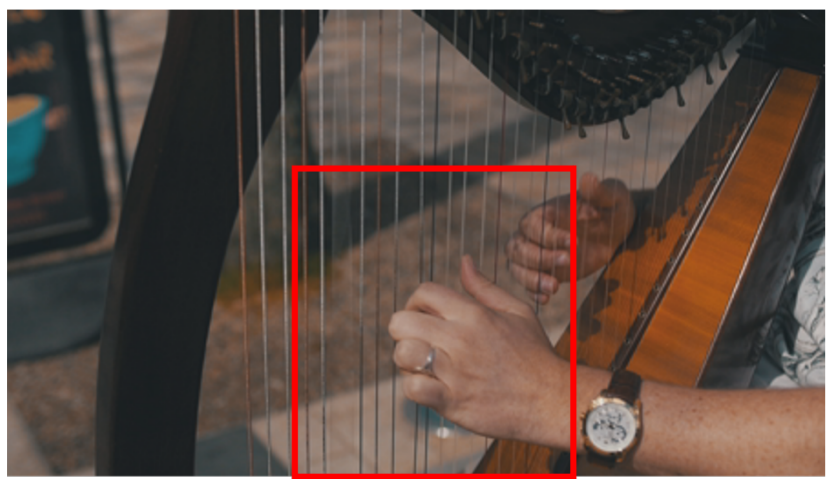

(a) Original

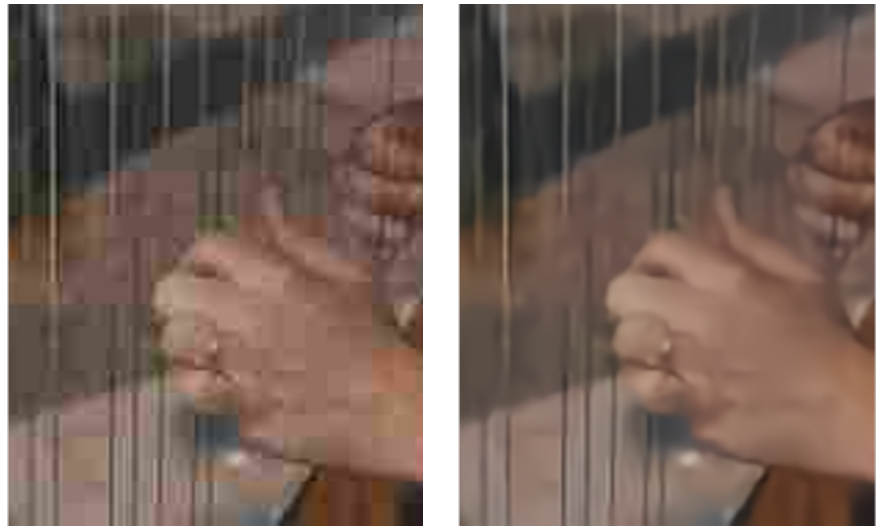

(b) Left: Compressed frame $(Q=15)$. Right: Deblocked image (RGB PSNR: $32.11 \mathrm{~dB})$

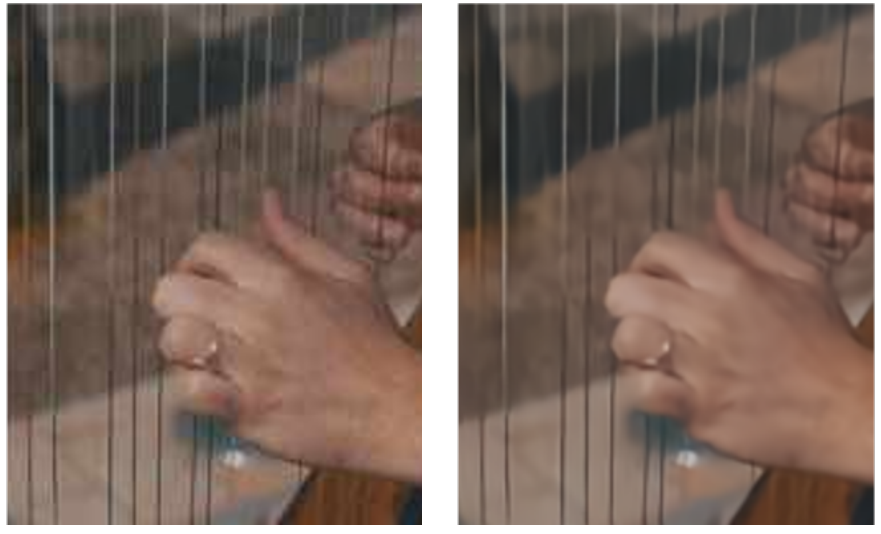

(c) Left: Compressed frame $(Q=45)$. Right: Deblocked image (RGB PSNR: $36.21 \mathrm{~dB})$
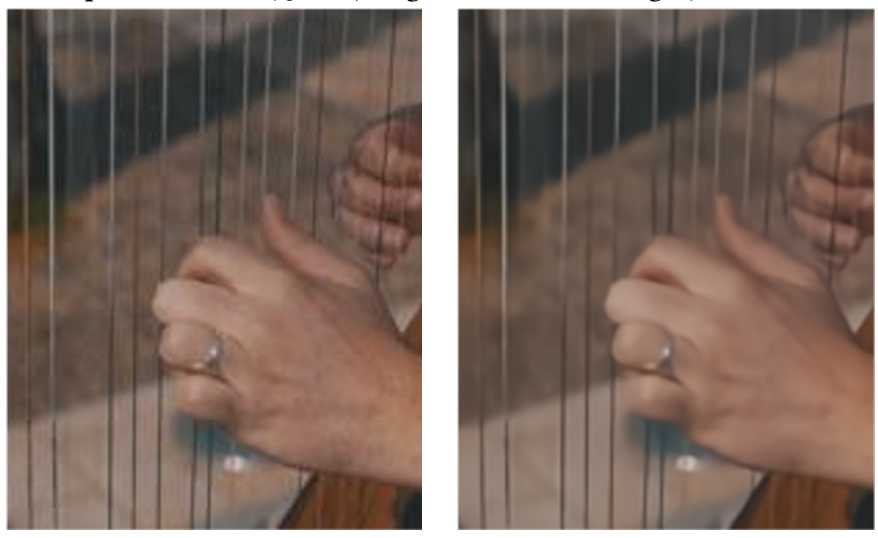

(d) Left: Compressed frame ( $Q=75)$. Right: Deblocked image (RGB PSNR: 37.56 dB)

Figure 2: Deblocking example at different values of $Q$. Note that lower value of $Q$ means higher compression. 


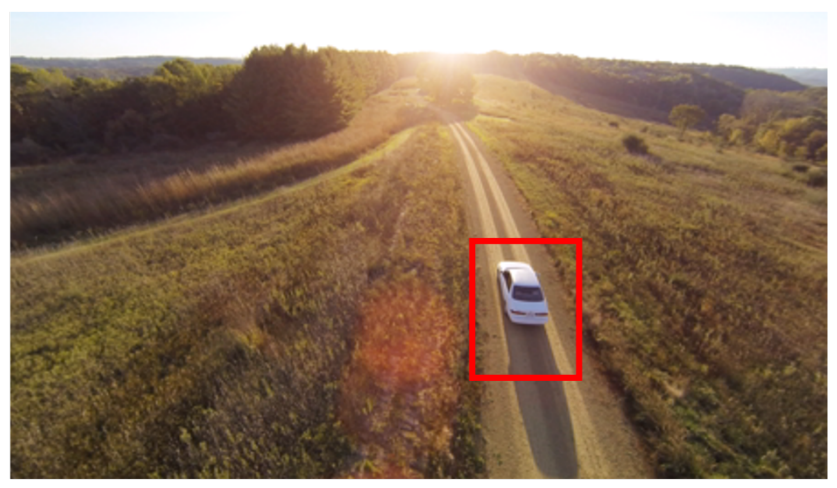

(a) Original
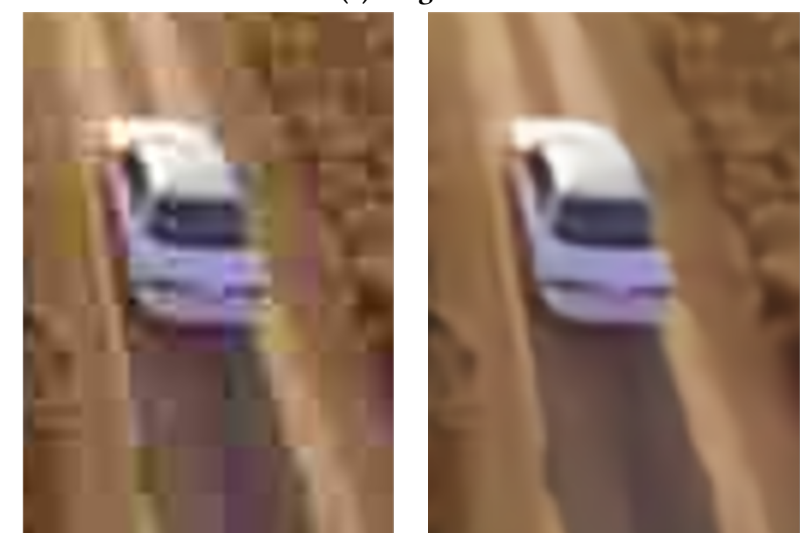

(b) Left: Compressed frame $(Q=15)$. Right: Deblocked image (RGB PSNR: $30.23 \mathrm{~dB})$
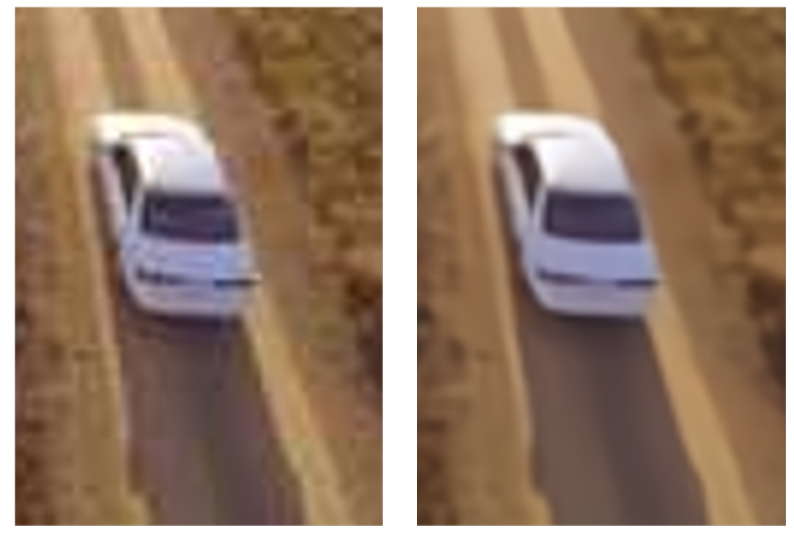

(c) Left: Compressed frame $(Q=45)$. Right: Deblocked image (RGB PSNR: $33.02 \mathrm{~dB})$
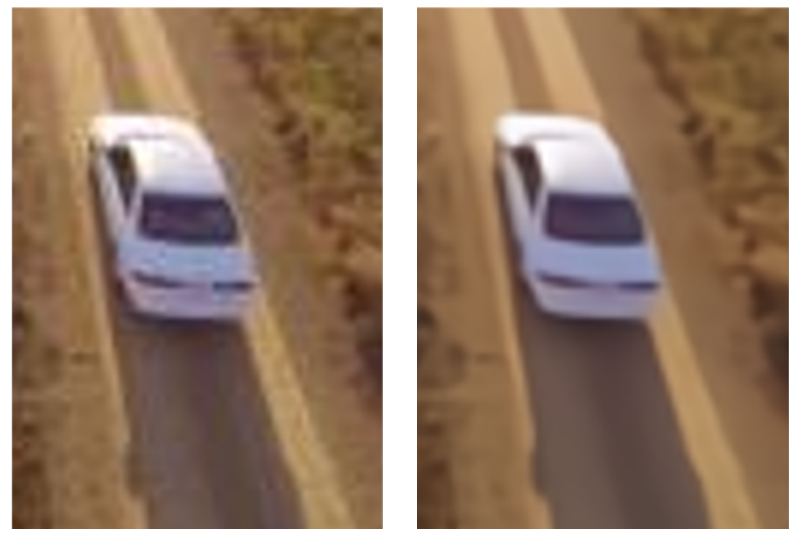

(d) Left: Compressed frame $(Q=75)$. Right: Deblocked image (RGB PSNR: $34.37 \mathrm{~dB})$

Figure 3: Deblocking example at different values of $Q$. Note that lower value of $Q$ means higher compression. 


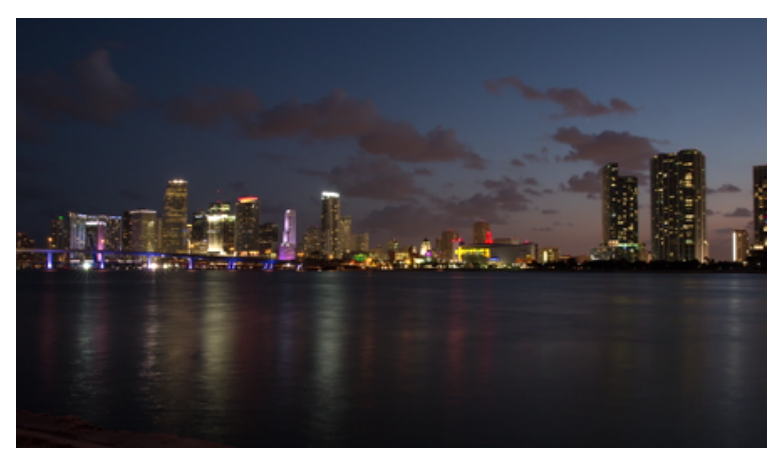

(a) Original

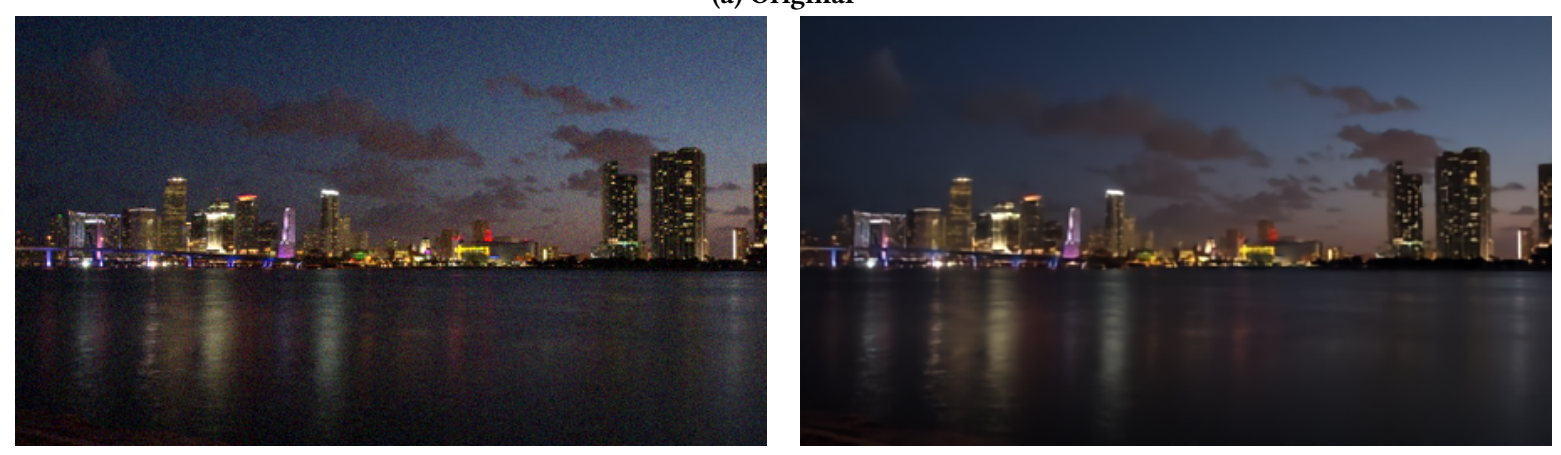

(b) Left: Noised image with AWGN $\left(\sigma^{2}=0.001\right)$. Right: Denoised image (RGB PSNR: $\left.34.57 \mathrm{~dB}\right)$

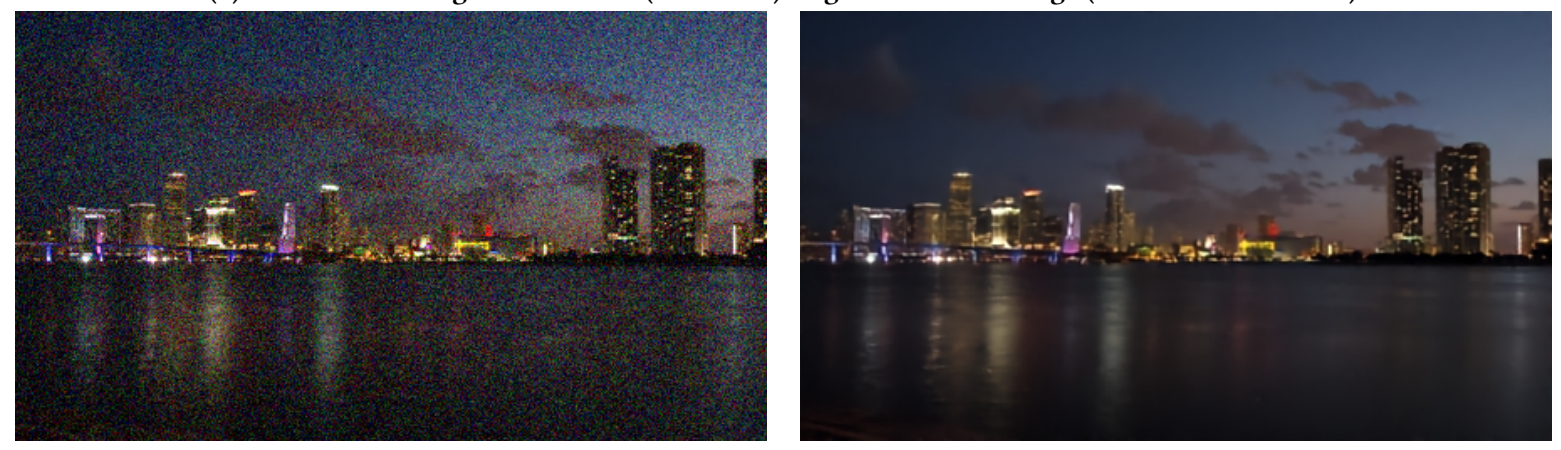

(c) Left: Noised image with AWGN $\left(\sigma^{2}=0.01\right)$. Right: Denoised image (RGB PSNR: 33.94 dB)

Figure 4: AWGN Denoising Example 


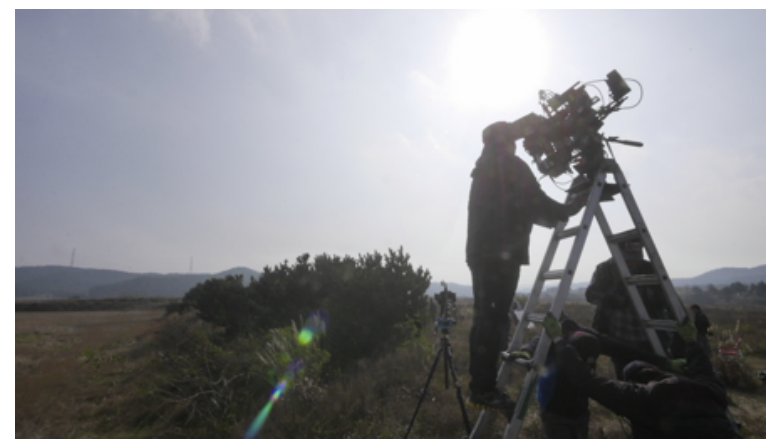

(a) Original
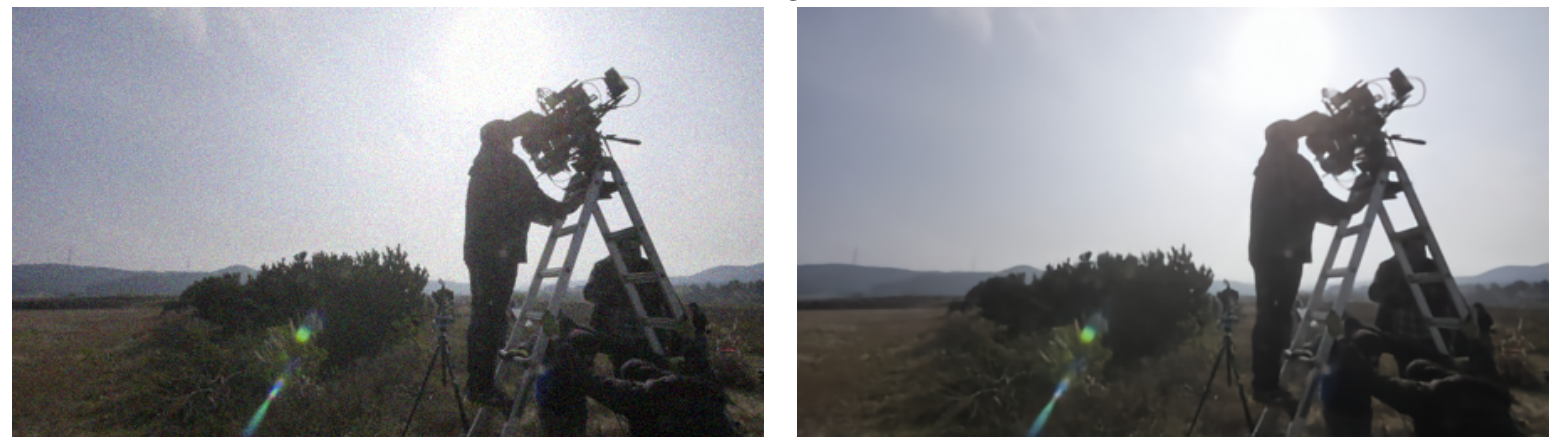

(b) Left: Noised image with AWGN $\left(\sigma^{2}=0.001\right)$. Right: Denoised image (RGB PSNR: $\left.38.67 \mathrm{~dB}\right)$
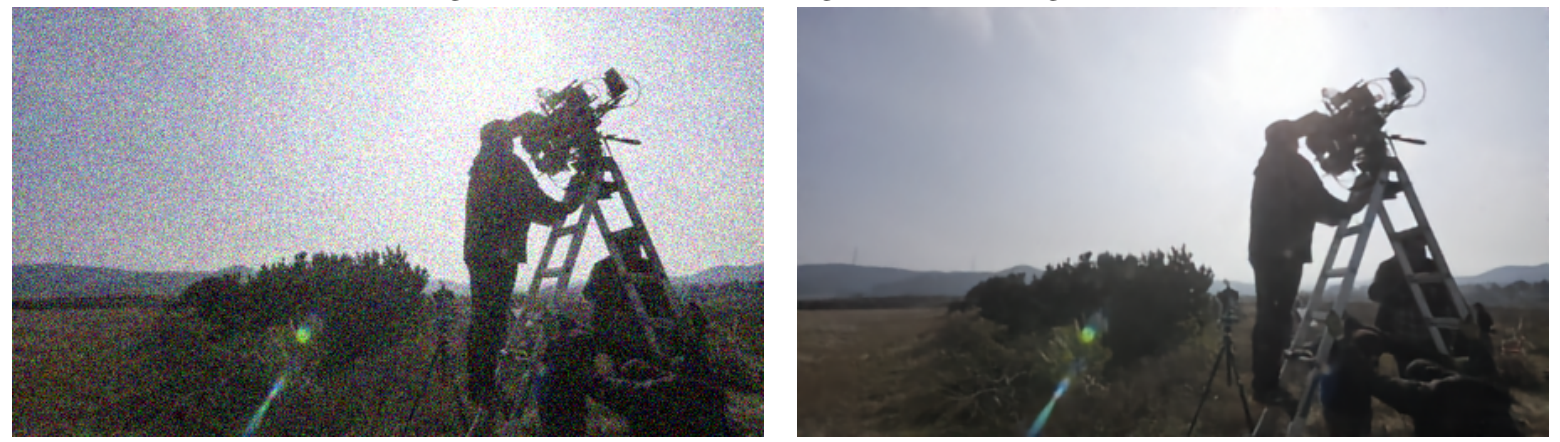

(c) Left: Noised image with AWGN $\left(\sigma^{2}=0.01\right)$. Right: Denoised image (RGB PSNR: $\left.37.11 \mathrm{~dB}\right)$

Figure 5: AWGN Denoising Example 


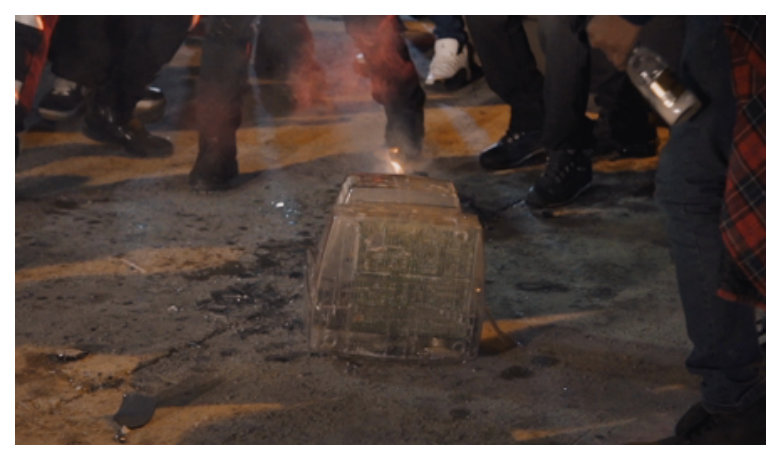

(a) Original
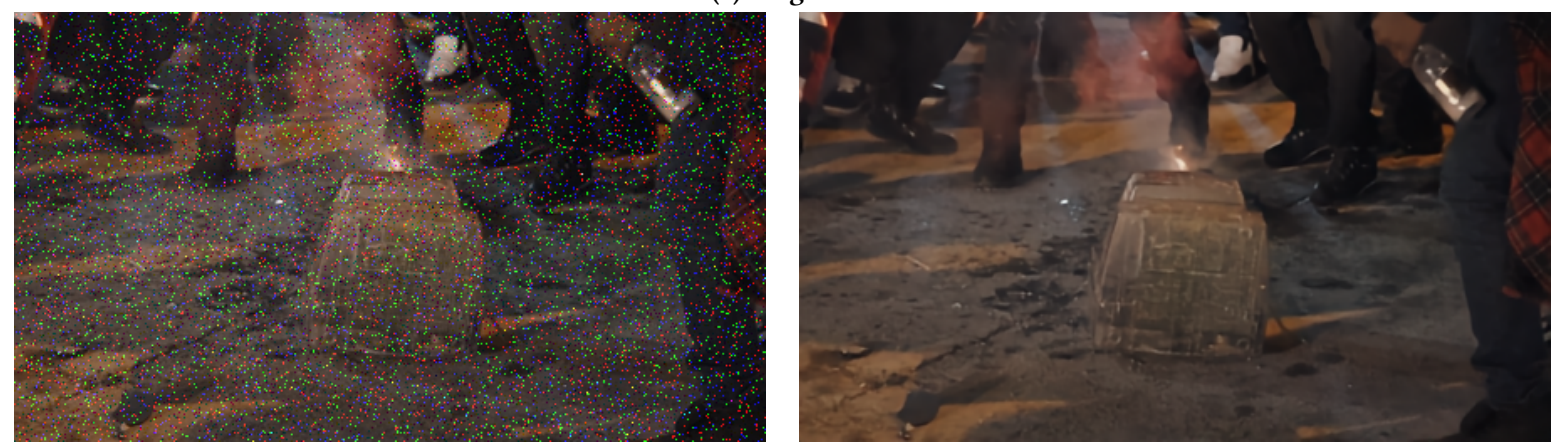

(b) Left: Noised image with S\&P $(\rho=0.05)$. Right: Denoised image (RGB PSNR: $38.17 \mathrm{~dB}$ )
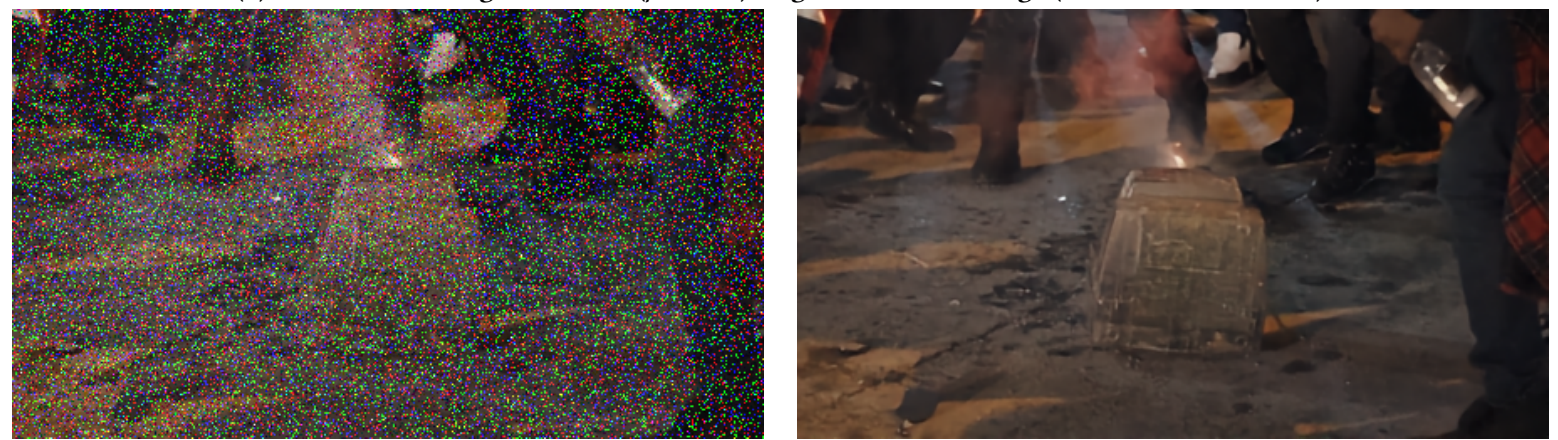

(c) Left: Noised image with S\&P $(\rho=0.15)$. Right: Denoised image (RGB PSNR: $37.28 \mathrm{~dB})$

Figure 6: Salt \& Pepper Denoising Example 


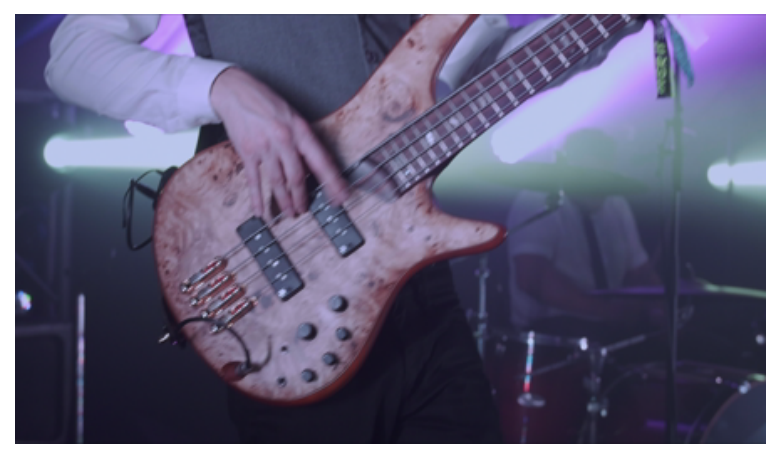

(a) Original
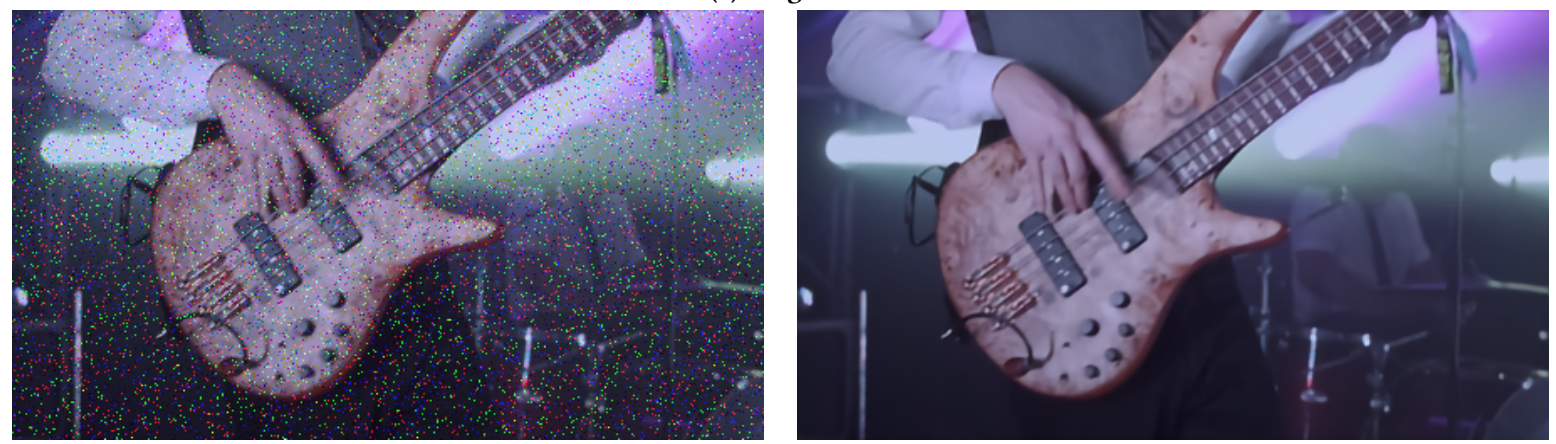

(b) Left: Noised image with S\&P $(\rho=0.05)$. Right: Denoised image (RGB PSNR: $36.30 \mathrm{~dB})$
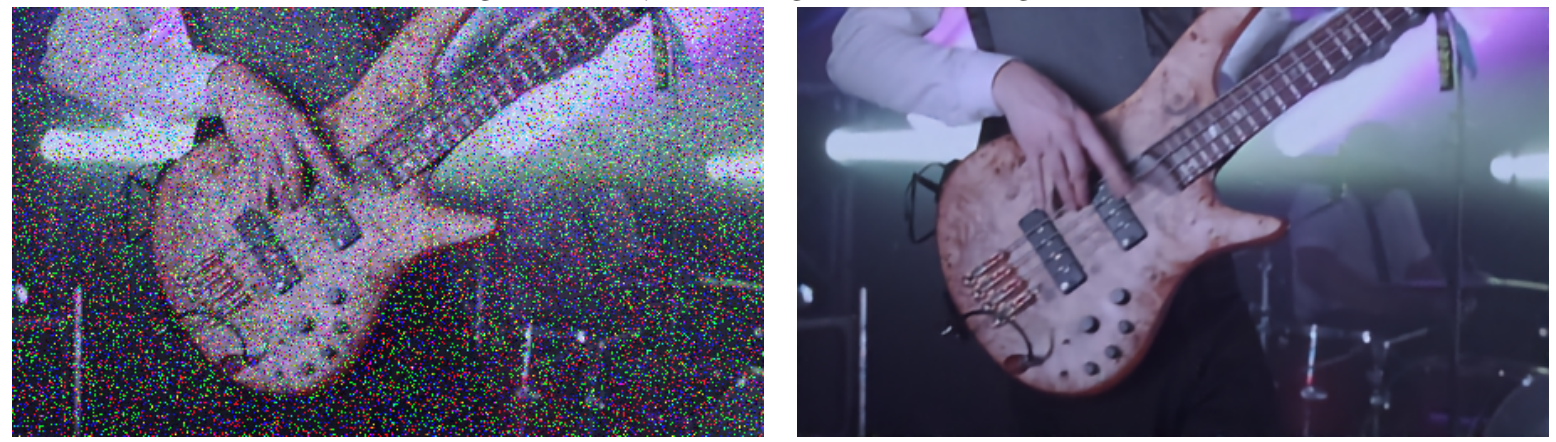

(c) Left: Noised image with S\&P $(\rho=0.15)$. Right: Denoised image (RGB PSNR: $35.50 \mathrm{~dB})$

Figure 7: Salt \& Pepper Denoising Example 

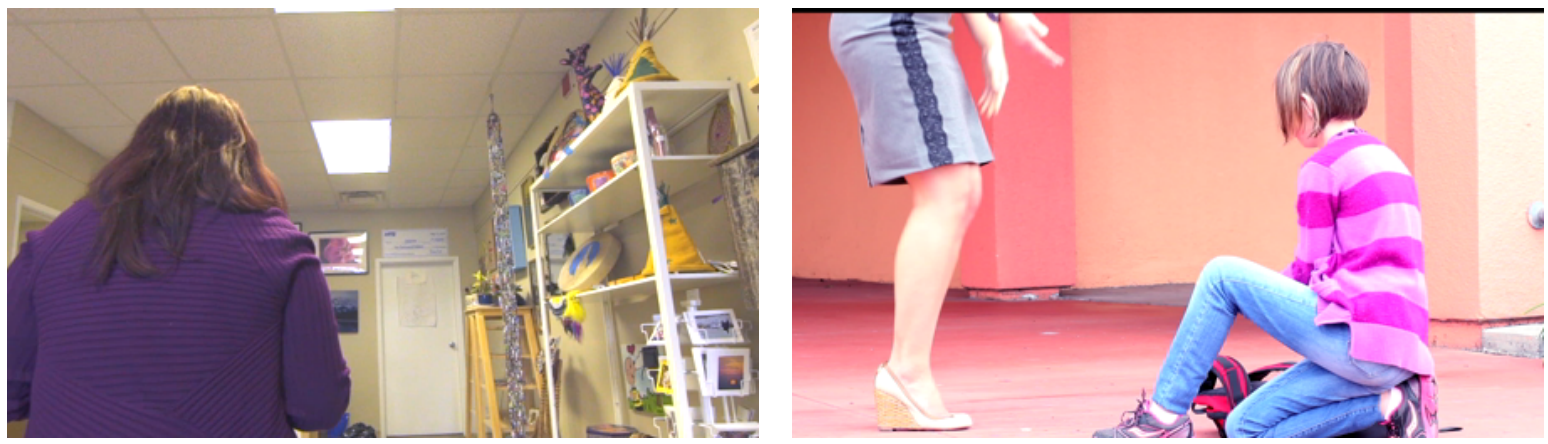

(a) Original images
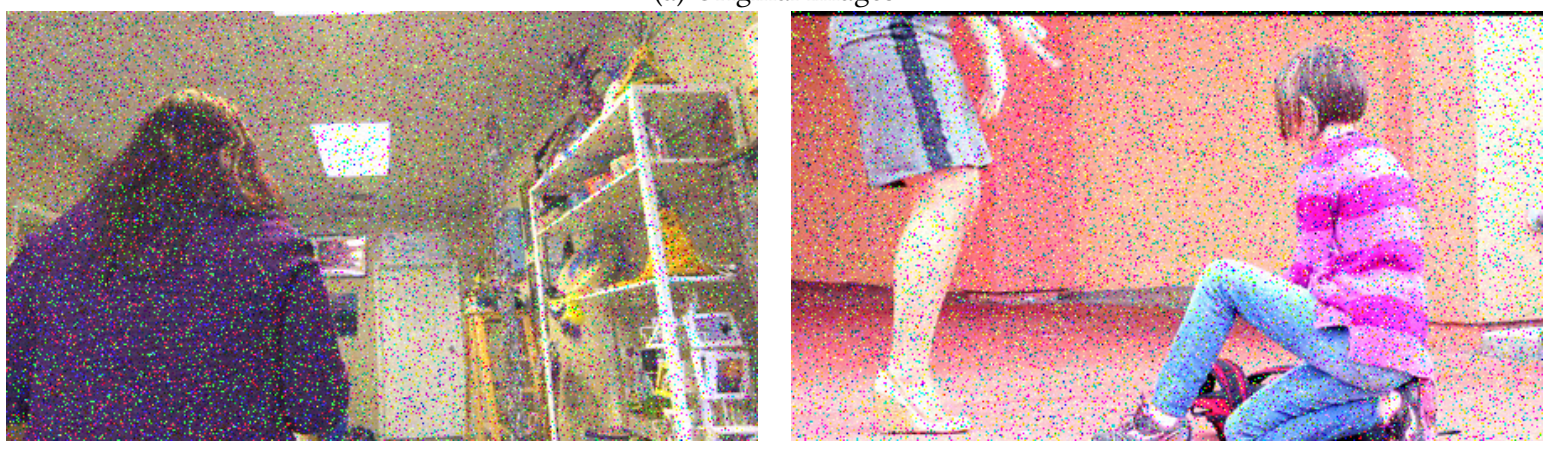

(b) Noised images with AWGN $\left(\sigma^{2}=0.001\right)$ and S\&P $(\rho=0.1)$
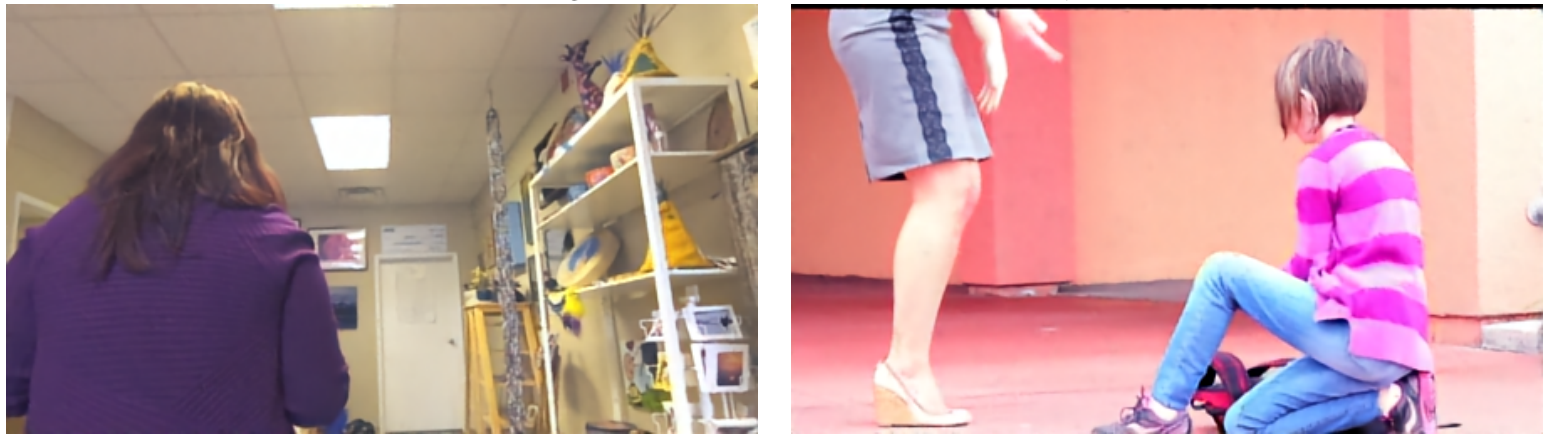

(c) Denoised images

Figure 8: Denoising example with mixed noise 

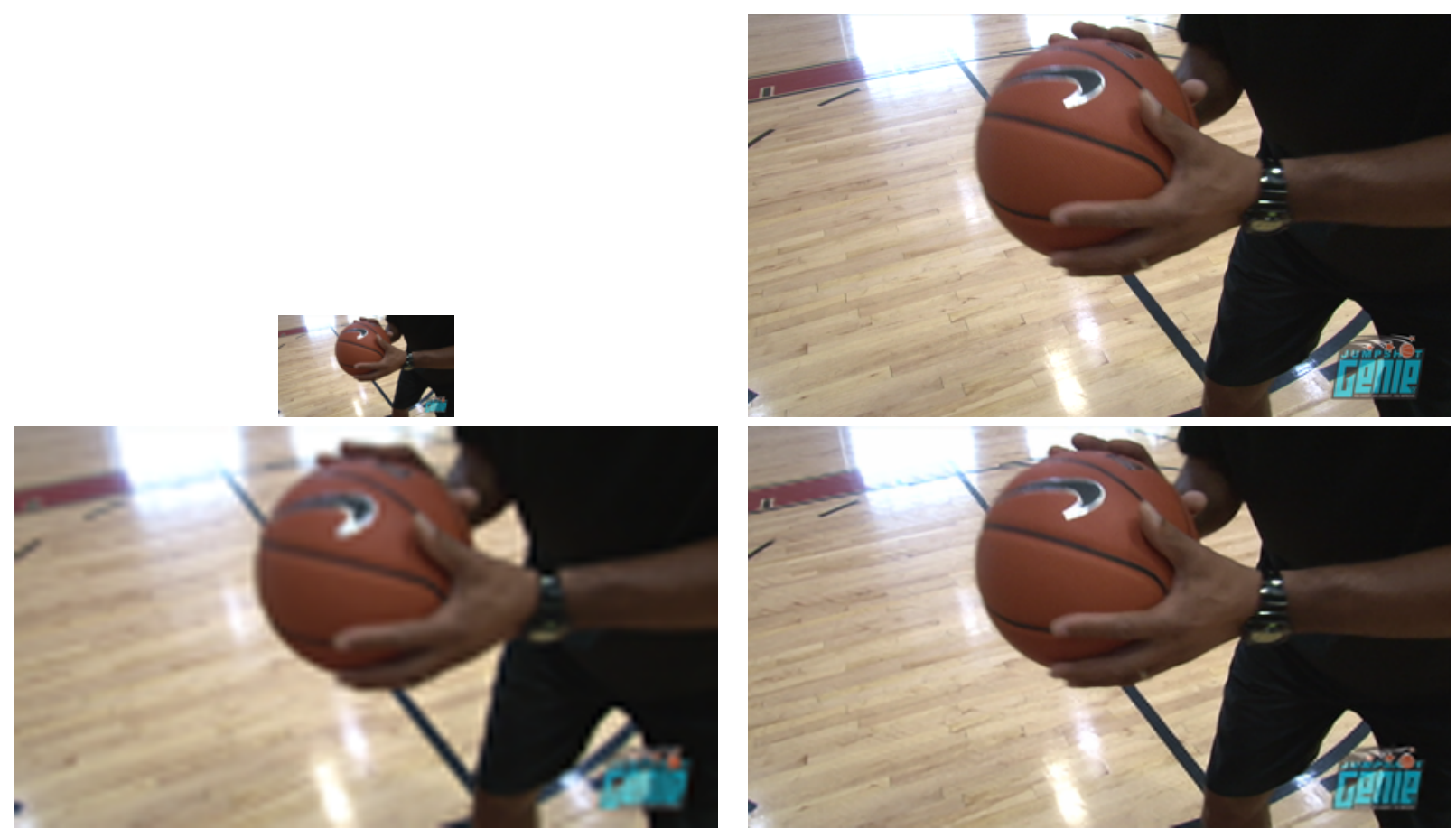

(a) Top left: Input low-resolution frame. Top right: Ground truth. Bottom left: Output of bicubic up-sampling (RGB PSNR: 28.59 dB) Bottom right: Output of EVRNet (RGB PSNR=34.76 dB).
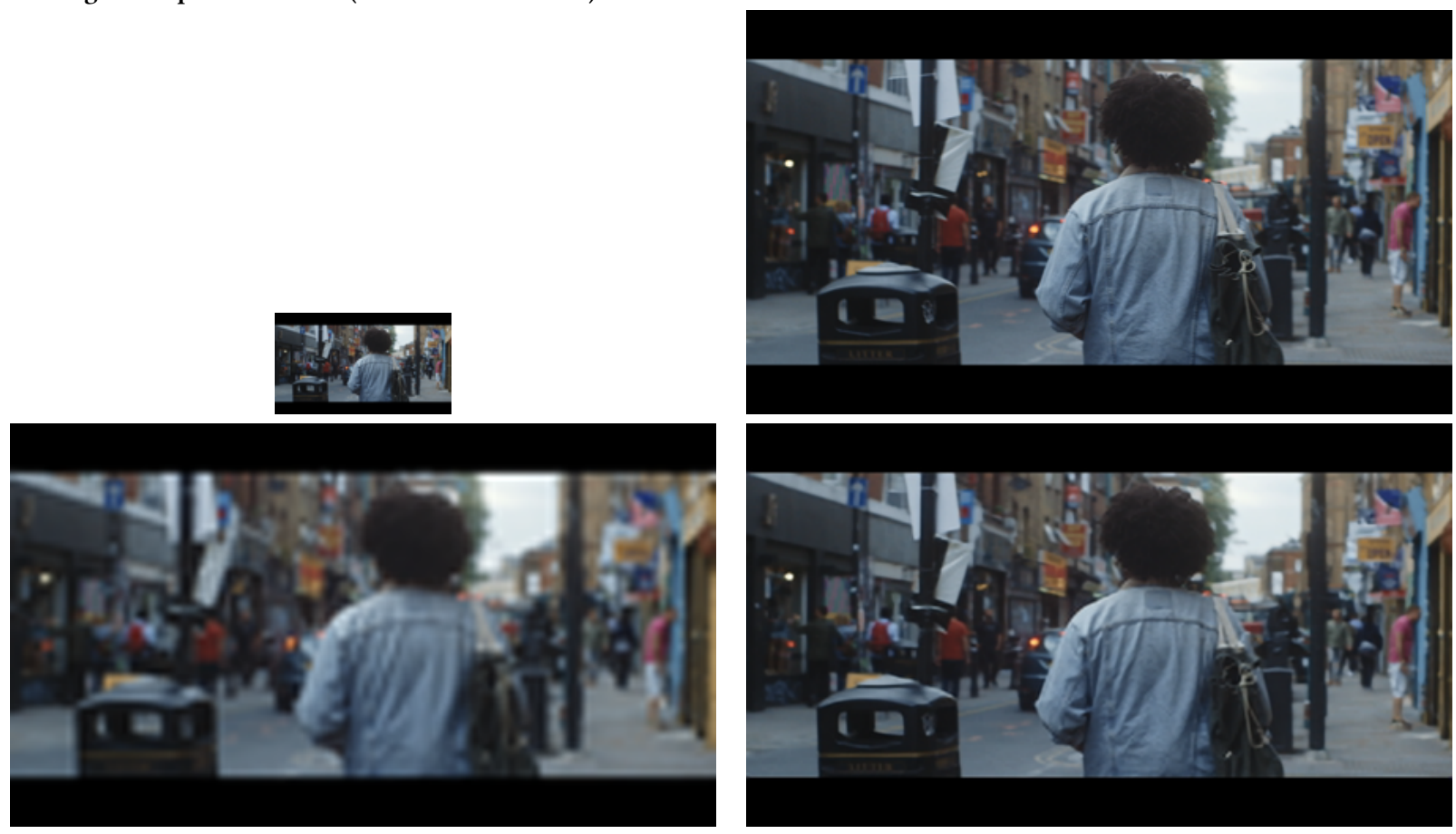

(b) Top left: Input low-resolution frame. Top right: Ground truth. Bottom left: Output of bicubic up-sampling (RGB PSNR: 27.56 dB) Bottom right: Output of EVRNet (RGB PSNR=38.41 dB).

Figure 9: $4 \times$ Video super-resolution examples. 


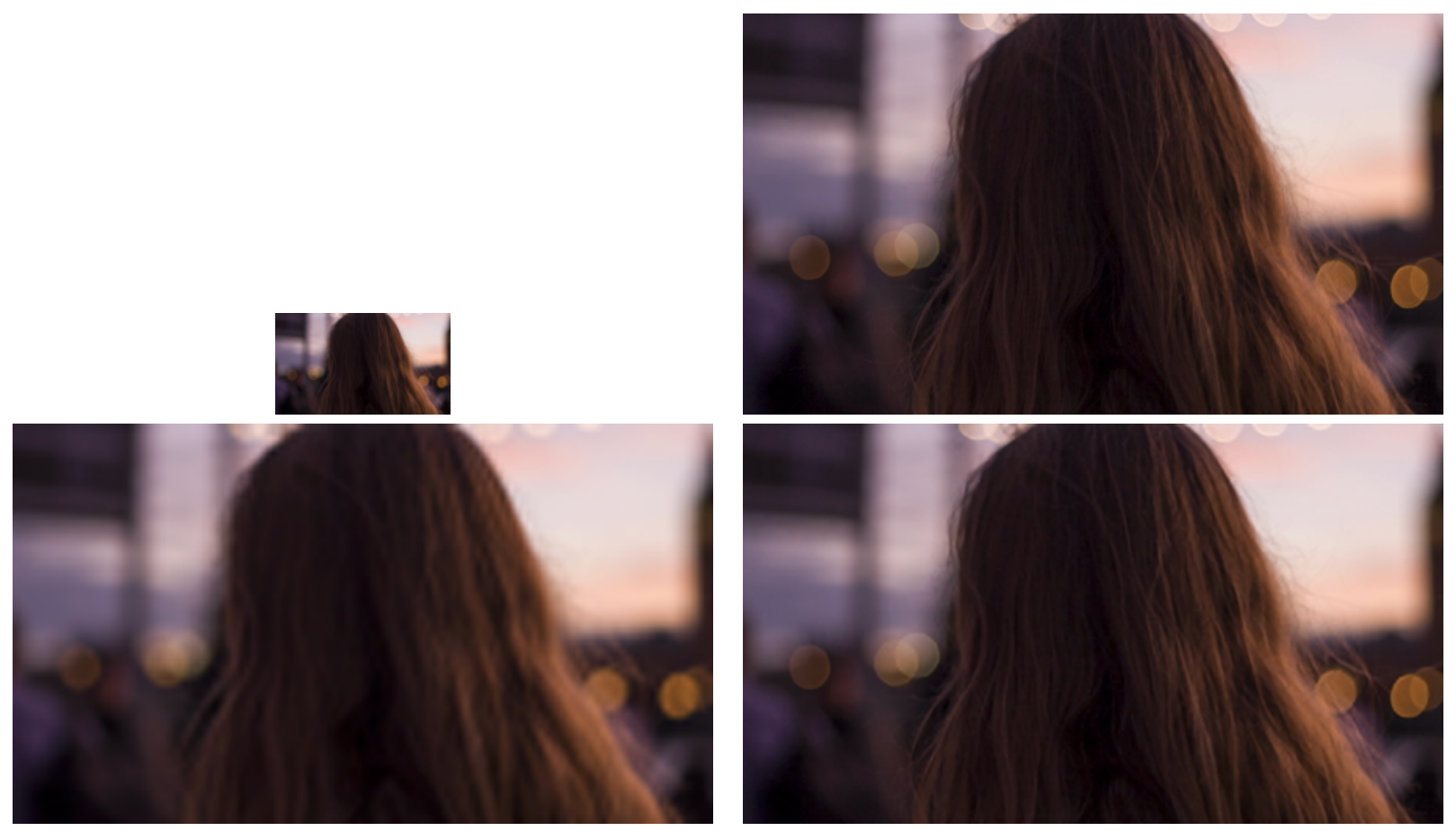

(a) Top left: Input low-resolution frame. Top right: Ground truth. Bottom left: Output of bicubic up-sampling (RGB PSNR: 36.84 dB) Bottom right: Output of EVRNet (RGB PSNR=42.84 dB).
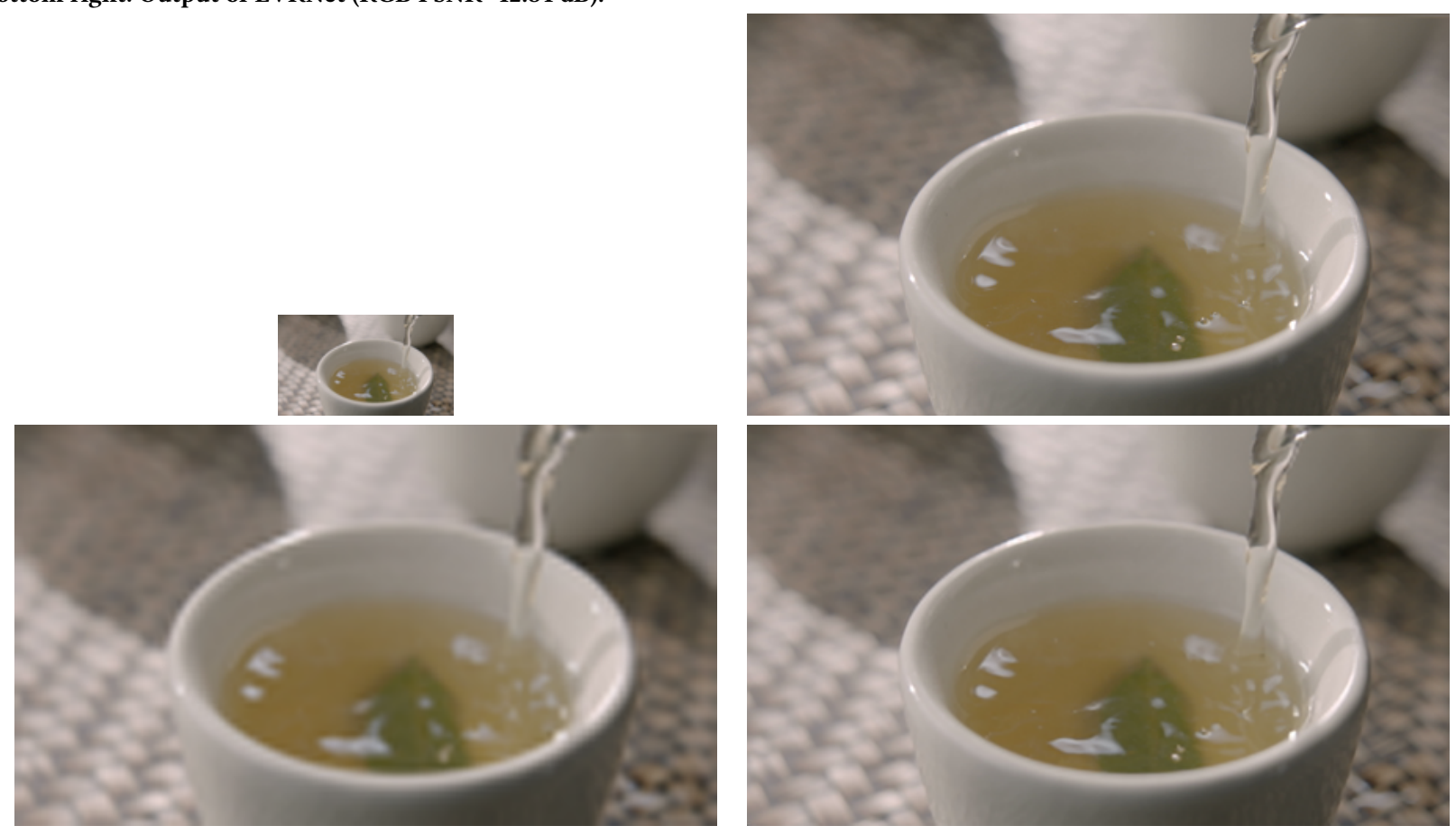

(b) Top left: Input low-resolution frame. Top right: Ground truth. Bottom left: Output of bicubic up-sampling (RGB PSNR: $36.21 \mathrm{~dB}$ ) Bottom right: Output of EVRNet (RGB PSNR=43.97 dB).

Figure 10: $4 \times$ Video super-resolution examples. 